\title{
The Circular Economy in the Popular Republic of China: A Multilevel Strategy for a New Model of Economic and Social Development
}

\section{Gianluca Senatore}

\author{
Sapienza Università di Roma, \\ Piazzale Aldo Moro, 5, 00185 \\ Roma RM, Italy
}

DOI: https://doi.org/10.36941/mjss-2021-0050

Abstract

The following work analyzes the principal differences in the implementation of the circular economy model between Europe and China, with a focus on practices and strategies set in place by the asiatic Popular Republic to promote the sustainable development. For that purpose, in this work, relevant trial studies on the management and deployment of circular economy in China and on green behaviors of Chinese population have been examined.

Keywords: China, sustainability, circular economy, development, environment strategy

\section{Economia Circolare in Europa}

Il concetto di economia circolare non ha tuttora una definizione puntuale ed operativa, anche se recenti studi (Kirchherr, Reike and Hekkert, 2017) sono giunti alla conclusione che il concetto alla base dell'idea della circular economy (CE) deve essere rappresentato da una struttura normativa che permette agli attori istituzionali di seguire determinati obiettivi: la sostenibilità ambientale, la prosperità economica e l'equità sociale. La circular economy viene quindi definita come «un sistema economico che sostituisce il concetto di 'fine vita' con la riduzione, il riutilizzo alternativo, il riciclaggio e il recupero dei materiali nei processi di produzione, distribuzione e consumo» (Kirchherr, Reike and Hekkert, 2017, p. 229).

Nell'ultimo decennio, l'economia circolare è stata posta al centro delle politiche europee in seguito alle preoccupazioni relative all'alto livello dei prezzi delle materie prime. In particolare, $\mathrm{i}$ primi passi sono stati mossi dai paesi dell'Europa nordoccidentale: Danimarca, Paesi Bassi, Germania e Regno Unito, che hanno, infatti, assunto un ruolo guida per l'introduzione di politiche sul riutilizzo dei materiali (Reike, 2018) anche se, alcune considerazioni in ambito europeo, alla luce degli ultimi dati, sono d'obbligo (Senatore and Teofili, 2021). L'UE ha definito obiettivi e direttive comuni a tutti gli stati membri al fine di definire politiche ed economie circolari, con la direttiva quadro sui rifiuti del 2008 (European Commission, 2008). In questo quadro normativo sono state introdotte misure specifiche sulla responsabilità estesa del produttore di rifiuti, politiche di gestione dei rifiuti, inquadramento normativo dei differenti rifiuti e relativa definizione, il riciclaggio, politiche di recupero e la definizione di una gerarchia dei rifiuti (Senatore and Teofili, 2021). Successivamente 
sono state annunciate una serie di misure di indirizzo politico più specifico, come il documento del 2015, "L'anello mancante: Piano d'azione dell'Unione Europea per l'economia circolare" (European Commission, 2015). In questo ambito vengono proposte delle modifiche alle direttive europee precedenti, attraverso la definizione di un quadro più sistemico (McDowall et al., 2017). Alla luce di questi indirizzi, la Commissione europea ha completato un processo di avvicinamento all'economia circolare che era iniziato negli anni precedenti con la definizione di ulteriori normative. Gli elementi chiave del piano d'azione per l'economia circolare riguardano la produzione, il consumo, la gestione dei rifiuti, i mercati dei materiali secondari nonché investimenti e innovazioni che favorissero un'economia circolare (McDowall et al., 2017). Nello specifico, il Piano d'azione definisce 54 misure finalizzate a "chiudere il cerchio", che comprendono cinque diverse aree di applicazione: i prodotti, con incentivi economici che spingessero i produttori a fornire prodotti più ecologici, durevoli e facilmente riciclabili, promuovendo quindi il loro recupero; la produzione, incentivando il riutilizzo dei materiali e promuovendo processi innovativi; il consumo, per garantire al consumatore la scelta di prodotti più naturali e sostenibili sono state introdotte misure volte al rispetto dei criteri giuridici e di affidabilità delle etichette verdi, inoltre è stata incoraggiata la condivisione di prodotti o infrastrutture, meglio conosciuta come sharing economy; la gestione dei rifiuti, che propone la revisione di sei direttive sui rifiuti, come la Direttiva quadro 2008/98/CE e l'introduzione di obiettivi di riciclaggio a lungo termine per rifiuti urbani e rifiuti di imballaggio; il mercato delle materie prime seconde, al fine di utilizzare materiali riciclati piuttosto che nuova materia vergine (European Commission, 2015).

Un più recente aggiornamento nel settore risale poi a luglio 2018, con l'introduzione del "pacchetto economia circolare", che prevede l'introduzione di quattro nuove direttive riguardo i rifiuti in generale, le discariche, gli imballaggi e i relativi rifiuti, le pile e i rifiuti di apparecchiature elettroniche ed elettriche (Senatore and Teofili, 2021). L'obiettivo principale degli Stati membri negli ultimi anni, quindi è stato quello di attuare misure che potessero prevenire soprattutto un eccessivo ricorso all'utilizzo di materie prime e quindi uno sfruttamento dell'ambiente (Feliziani, 2014), mentre prima gli impegni presi erano concentrati più sullo smaltimento dei rifiuti in discariche o inceneritori.

La politica europea sembra dunque determinata a proseguire in modo sempre più deciso verso un modello di green and circular economy, basato sui principi dello sviluppo sostenibile e dell'efficienza ecologica (Senatore and Teofili, 2021). A questo proposito, a dicembre 2019 è stato presentato dalla Commissione Europea il Green Deal, un ambizioso piano in cui viene espressa l'intenzione di fare dell'Europa il primo continente climaticamente neutrale entro il 2050, assicurando dunque una transizione ecologica che sappia garantire cicli di produzione rispettosi dell'ambiente (European Commission, 2019).

\section{Economia Circolare in Cina}

L'economia circolare in Cina si è sviluppata attraverso un percorso diverso rispetto a quello europeo. La notevole crescita economica della Cina degli ultimi decenni, che può essere rintracciata a partire dal 1978, con l'arrivo di Deng Xiaoping e le riforme che ne hanno definito la struttura economica ed industriale della Cina moderna, si concretizza con l'apertura al mercato estero, agli scambi, e alla delocalizzazione di aziende occidentali in territori cinesi. Gli straordinari incrementi di crescita industriale ed economica della Cina moderna hanno imposto reali e costanti cambiamenti nel tradizionale approvvigionamento delle materie prime e nel trattamento dei rifiuti. L'obiettivo principale della $C E$, nel caso della Cina, è stato gradualmente spostato da un riciclo limitato dei rifiuti ad un elevato controllo di tutte le fasi di produzione, distribuzione e consumo dei prodotti. In particolare, sono state trattate diverse aree, che spaziavano dai problemi delle risorse e dei rifiuti all'efficienza e la conservazione dell'energia, dalla gestione del territorio e la protezione del suolo alla gestione integrata delle risorse idriche (Su et al., 2013).

Nel tempo il concetto di CE della Cina è stato assimilato al concetto di ecologia industriale, che 
evidenzia i vantaggi dell'utilizzo di materiali di scarto e residui, ad esempio per la produzione di energia o il riutilizzo dell'acqua (Yuan et al., 2006). I benefici collettivi di tale visione ecologica risiedono sia in aspetti economici che ambientali (Anderson, 1994). Nella pianificazione di questa nuova visione ecologica industriale, la Cina riesce a comprendere quali possono essere i potenziali vantaggi nel riutilizzo dei materiali. Il passaggio a questa nuova visione avrebbe portato cambiamenti importanti al classico modello di sviluppo, coinvolgendo i tre aspetti fondamentali della sostenibilità, ovvero quello economico, sociale ed ambientale ( $\mathrm{Su}$ et al., 2013). Nel primo caso i vantaggi si sarebbero avuti grazie ad una maggiore competitività regionale e nazionale, dovuta ad un aumento dell'efficacia dell'allocazione delle risorse e del loro utilizzo e alla produttività. I vantaggi sociali si sarebbero avuti invece perché tale modello poteva creare opportunità di lavoro e permettere una crescita economica egualitaria, migliorando il benessere generale delle persone. Infine, dal punto di vista ambientale i vantaggi si sarebbero avuti in quanto poteva ridurre gli effetti negativi del modello tradizionale di produzione, riprogettando la struttura industriale in modo ecologico (Su et al., 2013).

Al fine di implementare la CE vennero introdotti all'interno della produzione e del consumo i principi delle ${ }_{3} R$ : Riduzione, Riuso e Riciclo. Con la riduzione si cercò di minimizzare l'utilizzo di energia primaria e materie prime, attraverso un miglioramento dell'efficienza produttiva. Con il riuso si intendeva invece riutilizzare i sottoprodotti e gli scarti di un'azienda come risorse per altre, in tal modo i prodotti avrebbero potuto essere utilizzati al massimo della loro capacità. Infine, con il riciclo si incoraggiava la trasformazione dei materiali, che potevano essere sottoposti a riciclo, in nuovi prodotti, al fine di diminuire il consumo di materiali vergini (Su et al., 2013).

A causa della rapida industrializzazione e urbanizzazione la Cina si è trovata ad affrontare sfide ambientali importanti, in particolare, il degrado del suolo, la desertificazione e la deforestazione, nonché l'esaurimento dell'acqua, un inquinamento generale e la perdita di biodiversità. Questo scenario ha contribuito ad un'accelerazione della CE all'interno del paese, che ha costretto la Cina ad aggiornare in maniera costante i piani di sviluppo economico e amministrativo. Ulteriori motivi per cui questo modello economico si è posto alla base della crescita della Cina, risiedono nella carenza di risorse ed energie, che non riescono a soddisfare la crescente domanda e hanno, già in passato, impedito la crescita economica del paese ( $\mathrm{Li}$ et al., 2010). La popolazione della Cina è pari, infatti, al $21 \%$ della popolazione mondiale, e possiede il $9 \%$ delle terre coltivate, con un $6 \%$ delle acque e il $4 \%$ delle foreste (Vermander, 2008), queste le risorse a disposizione per un così elevato numero della popolazione. A partire dal 2002, a seguito di una notevole crescita economica ed un'impennata della produzione da parte delle industrie pesanti, che ha determinato un enorme consumo delle risorse e delle energie (Su et al., 2013), il concetto di CE è stato formalmente accettato dal governo centrale come un'innovativa strategia di sviluppo. Il principale quadro nazionale cinese per perseguire la CE è stata dunque la Circular economy Promotion Law, entrata in vigore nel 2009, a cui sono seguiti diversi piani d'azione più dettagliati per settori specifici (McDowall et al., 2017).

Un percorso innovativo per uno sviluppo più sostenibile del paese è stato quindi necessario, e la CE è risultata essere una risoluzione per diversi motivi, anche per far fronte ai rigorosi standard e regolamenti ambientali in ambito internazionale, le cosiddette "barriere verdi", le quali possono frenare in maniera significativa le entrate commerciali dei paesi in via di sviluppo. Con l'attuazione della CE la Cina avrebbe potuto provvedere a questi obblighi commerciali, guadagnando una maggiore competitività nel commercio internazionale (Wang and Liu, 2007) (Su, 2013).

Da questo breve excursus si può notare quindi come, in generale, l'attuazione di un'economia circolare in Cina possa essere un intervento necessario per la risoluzione degli urgenti problemi di natura ambientale che il paese si trova ad affrontare e contemporaneamente un forte incentivo ad un nuovo modello economico in grado di soddisfare, almeno in parte, il bisogno di materie prime (Su et al., 2013). Per questo motivo tale strategia è stata implementata e ulteriormente sviluppata in diverse aree della Cina.

A differenza dell'Unione Europea, del Giappone o degli Stati Uniti, la CE in Cina si è sviluppata come un obiettivo politico nazionale, quindi, un modello di economia che non nasce da comportamenti individuali o collettivi indotti da particolari sensibilità dei cittadini, ma una strategia 
politica per puntare ad un maggiore sviluppo del paese e progettare politiche industriali efficienti economicamente e contestualmente sostenibili (Wang et al., 2018).

In particolare, lo sviluppo della CE in Cina è avvenuto in tre fasi (fig. 1) (Wang et al., 2018), partendo dalla prima fase che va dal 1996-98 al 2002, in un contesto in cui si evidenzia un forte aumento della produttività e dell'applicazione di maggiori vincoli ambientali: lo Stato inizia a sostenere i concetti di CE, con diverse normative come quella che regola la gestione del riciclaggio delle autovetture. In questa prima fase sono state poste le basi per una legislazione economica circolare più completa. Dopo il 2002, viene promulgata la "Legge per la promozione di una produzione più pulita" (2002-2009), che segna l'inizio di una fase d'azione della CE in Cina. In questo periodo lo stato emana infatti, una serie di leggi, regolamenti e politiche di sostegno fiscale per accelerare lo sviluppo della CE. Infine, nell'ultima fase, è stata emanata la "Legge per la promozione dell'economia circolare", che dopo i risultati avuti con gli interventi precedenti ha visto la sua approvazione durante la quarta riunione del Comitato permanente dell'undicesimo Congresso nazionale del popolo cinese, ed è entrata in vigore a gennaio 2009 (Geng et al., 2012), dando il via ad una fase di promozione attiva dell'economia circolare cinese.

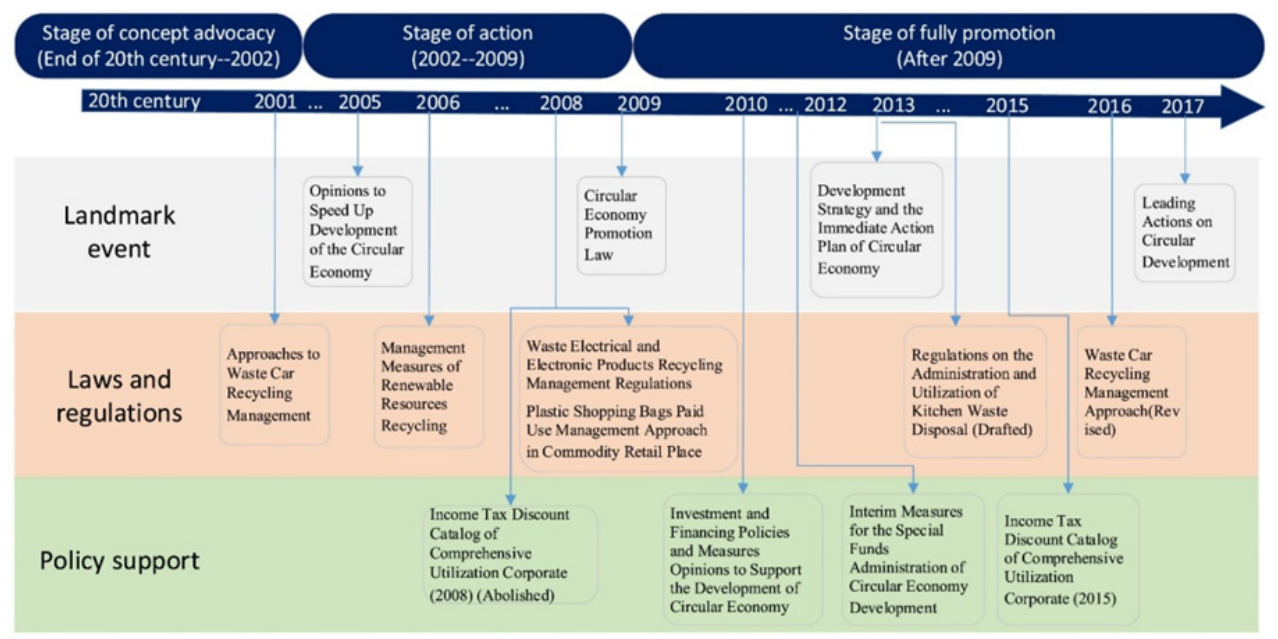

Figura 1: Sviluppo della CE in Cina

Fonte: Wang et al., 2018

\section{Pratiche di Economia Circolare in Cina}

Al fine di osservare ed analizzare quali sono state le azioni e le pratiche progettate ed attuate in Cina per concretizzare la $\mathrm{CE}$, in seguito alla presa di coscienza del paese sull'importanza di definire modelli economici di tipo non lineare, abbiamo voluto approfondire alcuni aspetti di questa particolare vicenda non solo economica, ma soprattutto sociale e culturale. Per approfondire il nostro studio, ci siamo concentrati su una ricerca che si è posta l'obiettivo di osservare in che modo è stata attuata la CE in Cina e con quale livello di coinvolgimento della popolazione, delle imprese e delle istituzioni locali e nazionali. Gli autori Su, Heshmati, Geng e Yu (2013) partono dalla considerazione che per attuare un buon livello di implementazione costante delle pratiche di CE è necessario agire su tre livelli dimensionali: micro-livelli, meso-livelli e macro-livelli (Su et al., 2013) (Yuan et al., 2006). Tali livelli sono integrati con quattro aree principali di attuazione delle pratiche: produzione, consumo, gestione dei rifiuti e altri supporti. La fig.2 è esemplificativa della struttura delle pratiche di CE attuate in Cina. 


\begin{tabular}{|c|c|c|c|}
\hline & Micro (single object) & $\begin{array}{l}\text { Meso (symbiosis } \\
\text { association) }\end{array}$ & $\begin{array}{l}\text { Macro (city, } \\
\text { province, state) }\end{array}$ \\
\hline $\begin{array}{l}\text { Production area(primary, } \\
\text { secondary, and tertiary } \\
\text { industry) }\end{array}$ & $\begin{array}{l}\text { Cleaner production } \\
\text { Eco-design }\end{array}$ & $\begin{array}{l}\text { Eco-industrial park } \\
\text { Eco-agricultural } \\
\text { system }\end{array}$ & $\begin{array}{l}\text { Regional eco- } \\
\text { industrial network }\end{array}$ \\
\hline Consumption area & $\begin{array}{l}\text { Green purchase and } \\
\text { consumption }\end{array}$ & $\begin{array}{l}\text { Environmentally } \\
\text { friendly park }\end{array}$ & Renting service \\
\hline Waste management area & $\begin{array}{l}\text { Product recycle } \\
\text { system }\end{array}$ & $\begin{array}{l}\text { Waste trade market } \\
\text { Venous industrial } \\
\text { park }\end{array}$ & Urban symbiosis \\
\hline Other support & $\begin{array}{l}\text { Policies and laws; Info } \\
\text { NGOs }\end{array}$ & rmation platform; C & city-building; \\
\hline
\end{tabular}

Figura 2: Struttura delle pratiche CE in Cina

Fonte: Su et al., 2013

Dalla fig.2 emerge come a livello micro, nell'area di produzione, le fabbriche e i produttori agricoli siano stati incoraggiati a adottare pratiche che garantiscano una produzione più pulita ed una progettazione ecocompatibile. Tra le due la misura più efficace ed attuata è stata la produzione più pulita, soprattutto in seguito all'emanazione della "Legge per la promozione della produzione più pulita”, a gennaio 2003 (Geng et al. 2010). L'obiettivo di questa strategia è stato quello di affrontare l'inquinamento attraverso un uso efficiente delle risorse, in ogni fase del processo produttivo, soprattutto per le imprese altamente inquinanti, svolgendo un ruolo primario nel ridurre le conseguenze che tali industrie hanno sull'ambiente, a causa anche della loro intensità energetica. L'eco-design è inteso invece come un'integrazione sistematica degli aspetti ambientali nella progettazione del processo di produzione e nel prodotto finale. In tal modo le aziende inquinanti sono state incoraggiate a generare modalità di produzione più integrate, efficienti e sostenibili, grazie ad un design innovativo della linea di produzione, soprattutto per far fronte agli effetti ambientali che si hanno nella prima fase del processo di sviluppo di un prodotto. Altre pratiche sono poi il consumo e l'acquisto green e una gestione dei rifiuti che hanno favorito lo sviluppo di aziende che si occupano delle risorse di scarto sia dei produttori che dei consumatori (Su et al., 2013).

A livello medio invece, le pratiche attuate hanno previsto lo sviluppo di parchi eco-industriali e un sistema eco-agricolo, la progettazione ecocompatibile di parchi ecologici e la costruzione di un sistema di scambio di rifiuti e parchi industriali che si sono occupati del recupero delle risorse, con aziende di tecnologia ambientale in grado di produrre quindi prodotti rispettosi dell'ambiente. Per l'area della produzione, il parco eco-industriale puntava ad applicare un concetto di simbiosi industriale attraverso cui potesse avvenire una gestione cooperativa del flusso di risorse delle imprese, raggruppate geograficamente (Su et al., 2013). In un parco eco-industriale quindi le aziende condividevano infrastrutture e servizi comuni e commerciavano sottoprodotti industriali, come energia, calore, acque reflue e rifiuti di produzione, in questo modo si avrebbero potuto aiutare le aziende a ridurre la dipendenza da risorse primarie e i loro impatti ambientali. Allo stesso modo funziona il sistema eco-agricolo, che mirava invece ad utilizzare sottoprodotti e rifiuti di colture e bestiame. Nell'area di consumo il programma principale è stato invece la progettazione "verde" delle comunità residenziali, attuata al fine di ridurre il consumo di energia, acqua e suolo. Inoltre, è stata elaborata la progettazione di un sistema in cui le acque reflue domestiche e i rifiuti solidi potessero essere raccolti e riciclati facilmente, al fine di favorire un ambiente abitativo eco-compatibile. L'area della gestione dei rifiuti, infine, prevedeva la regolamentazione del commercio dei rifiuti e la costruzione di un parco in grado di aumentare la produttività e i vantaggi del recupero delle risorse 
(Su et al., 2013).

A livello macro, ovvero su scala cittadina o regionale, nell'area produttiva sono state definite reti cooperative più complesse, che comprendevano industrie e parchi industriali del settore primario, secondario e terziario. Inoltre, al fine di rispettare i principi delle $3 R$, sono state riprogettate e riorganizzate le infrastrutture e il layout industriale delle città in base alle sue caratteristiche regionali ed eliminate gradualmente le imprese altamente inquinanti, che si cercarono di sostituire con industrie ad alta tecnologia, come l'agricoltura biologica. Per l'area del consumo è stato proposto invece un sistema di noleggio, basato sull'economia dei servizi, che spostava il focus dalla vendita e dall'acquisto dei prodotti al loro solo utilizzo (Stahel, 1986). Nel settore della gestione dei rifiuti venne invece incoraggiata la simbiosi urbana, un'estensione della simbiosi industriale (Geng et al., 2010a) precedentemente spiegata, che si concretizzava con attività che includevano prodotti e attrezzature rispettosi dell'ambiente, analisi ambientali e utilizzo di rifiuti riciclati.

Infine, per l'applicazione di pratiche di CE vi sono altri supporti (fig.1) che includono iniziative governative e non governative, ad esempio, il governo cinese regola l'attuazione della CE attraverso due agenzie: il ministero della protezione ambientale (MEP) e la Commissione nazionale per lo sviluppo e le riforme (NDRC) (Su et al., 2013). Oltre agli interventi governativi e gli sforzi industriali, intervengono anche organizzazione ambientali non governative, che rappresentano un requisito fondamentale per il successo delle pratiche di CE, in quanto stimolano cambiamenti di atteggiamento in tutta la società, quindi educazione, informazione e incoraggiamento alla partecipazione attiva del pubblico, che possono aumentare la consapevolezza delle persone sul tema.

\section{Il Comportamento dei Consumatori Verdi in Cina}

In che modo la popolazione cinese ha cambiato i propri comportamenti nel quotidiano per favorire uno sviluppo sostenibile? Le tecnologie ambientali, le politiche economiche e le iniziative sociali sono infatti fondamentali per una sostenibilità economica, ma la loro efficacia si basa sui cambiamenti effettivi dei modelli di comportamento di consumo (Peattie, 2010). La rapida urbanizzazione e industrializzazione della Cina, ha avuto un impatto significativo sugli atteggiamenti e sul comportamento dei consumatori cinesi (Zhao et al., 2014). Al fine di evidenziare in che modo sono cambiati i comportamenti di consumo tra la popolazione cinese, riporteremo di seguito i risultati di uno studio condotto da Zhao et al., (2014), incentrato sugli aspetti che influenzano tali comportamenti. Gli autori hanno analizzato tre aspetti separatamente: acquisto, utilizzo e riciclaggio.

Il sondaggio è stato condotto a Qingdao, al sud della penisola di Shandong, un'area metropolitana che comprende cinque città e sette distretti urbani (Zhao et al., 2014). Il metodo di ricerca utilizzato è stato il questionario, composto da domande con risposte preselezionate, che prevedevano tre sezioni. La prima sezione si concentrava sulle informazioni in base al consumo green, la seconda parte cercava invece di indagare la conoscenza dei soggetti sul consumo, gli atteggiamenti nei confronti del consumo verde e il ruolo dei moderatori interni ed esterni. Per moderatori interni si intendono le principali preoccupazioni ambientali e la percezione del consumatore, secondo cui, ad esempio gli individui che dimostrano una maggiore preoccupazione ambientale hanno più probabilità di intraprendere azioni green; per moderatori esterni si intendono invece le azioni intraprese dai governi e le aziende. La terza sezione, infine, era finalizzata a raccogliere informazioni demografiche.

Tra i 500 intervistati il 48,6\% erano femmine e il 51,4\% maschi, per la maggior parte di età compresa tra i 31 e i 50 anni. Per quanto riguarda il livello di istruzione, gli intervistati avevano prevalentemente un'istruzione superiore $(24,4 \%)$, seguita da un livello universitario $(17,8 \%)$.

Entrando nello specifico della ricerca, è emerso che molti degli intervistati erano a conoscenza di cosa fosse il consumo green, 362 su 500 hanno infatti risposto di aver sentito parlare di consumo sostenibile. Per quanto riguarda invece le fonti di informazione su questo tipo di consumo, il canale principale era la tv, seguita da internet e dai giornali (fig.3). 


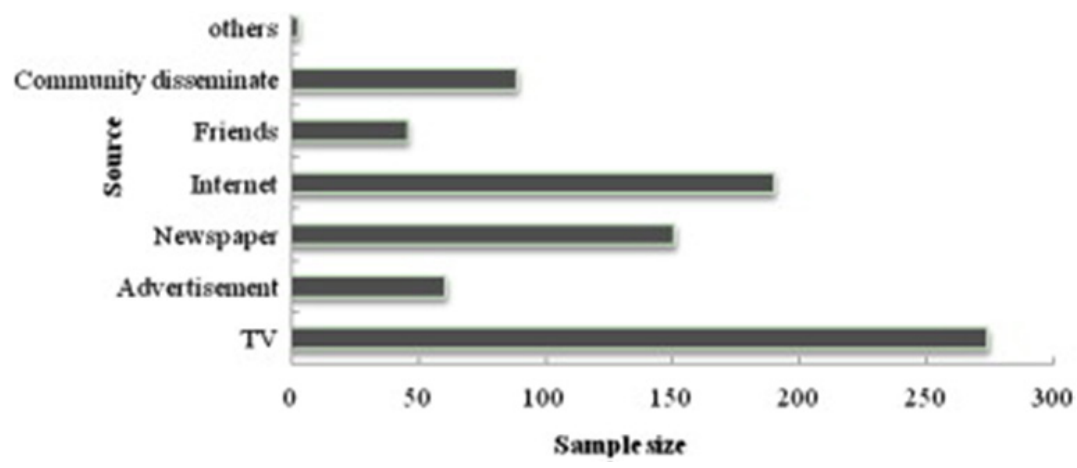

Figura 3: Canali di informazione sul consumo verde

Fonte: Zhao et al., 2014

Ulteriori domande poste agli intervistati riguardavano poi la disponibilità a pagare e il comportamento di acquisto, ed è emerso che il 71,6\% dei consumatori era disposto a pagare prodotti green anche ad un prezzo elevato, dimostrando come la maggior parte dei cittadini di Qingdao desiderasse fortemente sostenere finanziariamente i prodotti che rispettavano l'ambiente. Il $26,4 \%$ ha infatti affermato che avrebbe scelto prodotti sostenibili anche con un prezzo tra il $5 \%$ e il $10 \%$ più elevato rispetto ai prodotti convenzionali (fig.4).

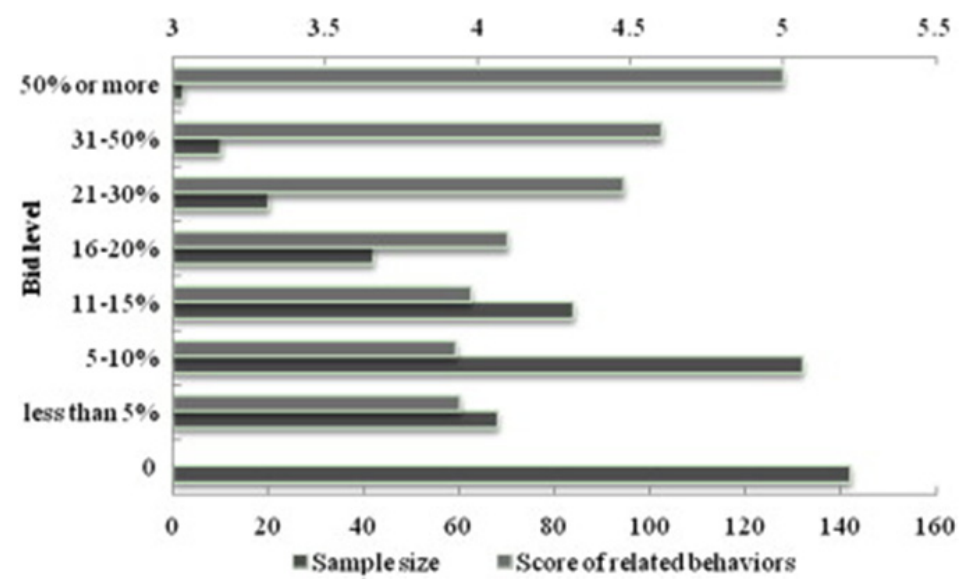

Figura 4: Disponibilità a pagare per prodotti verdi costosi e punteggio del comportamento di acquisto.

Fonte: Zhao et al., 2014

Riguardo i tre aspetti del consumo, l'acquisto, l'utilizzo e il riciclaggio, è emersa una notevole variazione tra le diverse attività. Tra i tre comportamenti, infatti, il riciclaggio è risultato quello più diffuso, mentre il meno diffuso è stato l'utilizzo. Il motivo può dipendere dal fatto che questi comportamenti hanno motivazioni intrinseche differenti (Wang, 2010). Ad esempio, l'acquisto comporta costi personali per consentire lo sviluppo di benefici pubblici, quindi i consumatori sono costretti a pagare di più per i prodotti che rispettano l'ambiente, oppure, l'aspetto dell'utilizzo implica la necessità di cambiare le abitudini di utilizzo dell'acqua nella vita quotidiana e ancora, il riciclaggio dipende dall'interesse privato e dato i vantaggi che in Cina si hanno per azioni come il riciclo di vecchi giornali o lattine, i consumatori mettono in pratica i loro comportamenti green 
soprattutto attraverso il riciclo, perché appunto ne ottengono vantaggi, molto spesso anche economici (Huang et al., 2006).

Un ulteriore aspetto rilevante, emerso dalla ricerca, è la relazione tra i dati demografici e altri costrutti del consumo green, in particolare i consumatori più istruiti tendono a dare rilevanza a tutti e tre gli aspetti, questo perché un maggiore livello di istruzione consente una migliore comprensione delle questioni ambientali, dunque questi soggetti sono più interessati alla qualità ambientale e disposti ad assumere un comportamento sostenibile. Per quanto riguarda invece la relazione tra età e comportamento dei consumatori ecologici, la ricerca ha dimostrato che la popolazione più anziana ha maggiori probabilità di adottare comportamenti quali riciclaggio e utilizzo, aspetto dovuto all'etica di conservazione di tale generazione, caratterizzata dall' "era della depressione" (Zhao et al., 2014). Il gruppo più anziano in Cina assume quindi comportamenti sostenibili come il riutilizzo dei sacchetti di carta durante la spesa o la conservazione dell'acqua, perché ha sviluppato un'abitudine al risparmio come risultato delle esperienze dell'era vissuta ed è più incentivato a intraprendere comportamenti di utilizzo e riciclaggio. Le abitudini di vita, infatti, influenzano maggiormente le attività quotidiane, più del pensiero cosciente (Carrus et al., 2008). Il reddito è correlato positivamente, anche se non in modo significativo, con il comportamento di acquisto, in quanto i prodotti sostenibili hanno generalmente un prezzo più alto rispetto a quelli convenzionali, dunque i gruppi con un reddito elevato potrebbero essere più propensi ad acquistare tali prodotti perché ne possono sostenere i costi. Infine, un ulteriore aspetto interessante è stata la relazione negativa tra reddito e comportamento di utilizzo e riciclaggio, aspetto dovuto soprattutto alla trasformazione della Cina in una società di consumo di massa. I consumatori cinesi con un reddito più elevato ricercano infatti migliori comfort e non sono disposti a mettere in secondo piano gli interessi personali per scopi e benefici collettivi, mostrando quindi una minore propensione ai comportamenti di utilizzo e riciclaggio.

\section{Conclusioni}

Il modello di economia circolare è risultato necessario in Cina, in particolare a causa dei gravi problemi ambientali che il paese si è trovato ad affrontare e per incentivare una strategia in grado di soddisfare, anche se solo in parte, il bisogno di materie prime ( $\mathrm{Su}$ et al., 2013). La visione di un approccio più sostenibile ed ecologico si è quindi sviluppata in maniera sempre maggiore tra la popolazione cinese, sia grazie agli interventi delle istituzioni, che hanno definito diverse politiche a favore di questo modello economico, sia grazie al supporto delle organizzazioni non governative, che si sono invece concentrate su aspetti altrettanto importanti per garantire un'efficace sviluppo sostenibile, ovvero l'informazione, l'educazione e l'incoraggiamento ad una partecipazione attiva del pubblico.

Dagli studi condotti sulle effettive pratiche attuate in Cina per favorire una trasformazione ecologica (Su et al., 2013), è emersa una suddivisione degli interventi in quattro aree principali: la produzione, il consumo, la gestione dei rifiuti ed altri supporti come le organizzazioni non governative. Tali interventi hanno poi coinvolto tre livelli di attuazione: a livello micro, comprendendo quindi le azioni dei singoli soggetti; a livello medio, includendo quindi le attività delle associazioni di persone; a livello macro, ovvero gli interventi provinciali o regionali. Per quanto riguarda invece i comportamenti green dei consumatori, le ricerche condotte hanno evidenziato una particolare preferenza per le attività di riciclaggio, soprattutto da parte delle generazioni più anziane, che hanno vissuto un'era di depressione (Zhao et al., 2014).

In conclusione, la sostenibilità in Cina è stato un concetto che si è cercato di mettere in pratica attraverso modelli di economia non lineari, con interventi che non sono nati dal basso, come è accaduto per altri paesi, ma sono stati imposti dal governo come risoluzione a diverse problematiche che il paese si è trovato ad affrontare. 


\section{Bibliografia}

Anderson G. (1994). Industry clustering for economic development. Economic Development Review. 12 (2): 26-32.

Carrus G., Passafaro P., Bonnes M. (2008). Emotions, habits and rational choices in ecological behaviours: The case of recycling and use of public transportation. Journal of Environmental Psychology. 28 (1): 51-62. Doi: 10.1016/j.jenvp.2007.09.003.

European Commission. (2008). Commissione Europea sulla gestione dei rifiuti. Testo disponibile all'indirizzo web: https://eur-lex.europa.eu/legal-content/IT/LSU/?uri=CELEX:32008Loo98

European Commission. (2015). Closing the loop: An action plan for the circular economy. Testo disponibile all'indirizzo web: https://eur-lex.europa.eu/legal-content/IT/TXT/?uri=CELEX:52015DCo614

European Commission. (2019). A European Green Deal. Testo disponibile all'indirizzo web: https://ec.europa.eu/info/strategy/priorities-2019-2024/european-green-deal_en

Feliziani C. (2014). Tutela Ambientale e servizio pubblico: il caso di gestione dei rifiuti in Italia e Inghilterra. Roma: Sapienza Università editrice.

Geng Y., Xinbei W., Qinghua Z., Hengxin Z. (2010). Regional initiatives on promoting cleaner production in China: a case of Liaoning. Journal of Cleaner Production. 18 (15): 1502-1508. Doi: 10.1016/j.jclepro.2010.06.o28.

Geng Y., Tsuyoshi F., Chen X. (2010a). Evaluation of innovative municipal solid waste management through urban symbiosis: a case study of Kawasaki. Journal of Cleaner Production. 18 (10-11): 993-10oo. Doi: 10.1016/j.jclepro.2010.03.003.

Geng Y., Fu J., Sarkis J., Xue B. (2012). Towards a national circular economy indicator system in China: an evaluation and critical analysis. Journal of Cleaner Production. 23 (1): 216-224. Doi: 10.1016/j.jclepro.2011.07.005.

Huang P., Zhang X., Deng X. (2006). Survey and analysis of public environmental awareness and performance in Ningbo, China: a case study on household electrical and electronic equipment. Journal of Cleaner Production. 14 (18): 1635-1643. Doi: 10.1016/j.jclepro.2006.02.006.

Kirchherr J., Reike D., Hekkert M., (2017). Conceptualizing the circular economy: An analysis of 114 definitions. Resources, Conservation and Recycling. Vol. 127, pp. 221-232. Doi: 10.1016/j.resconrec.2017.09.005

Li H., Bao W., Xiu C., Zhang Y., Xu H. (2010). Energy conservation and circular economy in China's process industries. Energy. 35 (11): 4273-4281. Doi: 10.1016/j.energy.2009.04.021

McDowall W., Geng Y., Huang B., Barteková E., Bleischwitz R., Türkeli S., Kemp R. and Doménech T. (2017). Circular economy Policies in China and Europe. Journal of Industrial Ecology. 21, pp. 651-661. Doi: 10.1111/jiec.12597

Peattie K. (2010). Green consumption: behavior and norms. Annual Review of Environment and Resources. 35 (1): 195-228. Doi: 10.1146/annurev-environ-032609-094328

Reike D., Vermeulen W.J.V., Witjes S. (2018). The circular economy: New or Refurbished as CE 3.0? - Exploring Controversies in the Conceptualization of the Circular economy through a Focus on History and Resource Value Retention Options. Resources, Conservation and Recycling. 135, pp. 246-264. Doi: 10.1016/j.resconrec.2017.08.027.

Senatore G. (2020). Culturalizzazione della società condizione necessaria per la sostenibilità. In Mantione G., Romanelli E., a cura di, Il corpo della terra. La relazione negata. Da una visione egologica a una visione ecologica. Roma: Castelvecchi Editore.

Senatore G., Teofili S. (2021). Waste and Circular economy. Mediterranean Journal of Social Science. Vol. 12, n.3. Doi: 10.36941/mjss-2021-0017

Stahel W.R. (1997). The functional economy: Cultural and organizational change, in D.J. Richards, a cura di, The Industrial Green Game: Implications for Environmental Design and Management. Washington D. C.: National Academy Press.

Su B., Heshmati A., Geng Y., Yu X., (2013). A review of the circular economy in China: moving from rhetoric to implementation. Journal of Cleaner Production. 42, 215-227. Doi: 10.1016/j.jclepro.2012.11.020.

Vermander, B. (2008). A growth engine reinvents itself: towards a greener China? In Elvire Fabry and Damien Tresallet, a cura di, Greening Economic Growth: Towards a Global Strategy for Europe. Parigi: Fondation pour l'innovation Politique.

Wang Y.J, Liu H.H. (2007). Green barriers from the standpoint of sustainable development. Journal of Economic Policy Reform. 10 (3): 233-240. Doi: 10.1080/17487870701456545

Wang, J. M. (2010). Consumers' Resource-saving \& Environment-protecting Behavior and Its Mechanism. Pechino: China Social Science Press.

Wang N., Lee J.C.K, Zhang J., Chen H., Li H. (2018). Evaluation of Urban circular economy development: An empirical research of 40 cities in China. Journal of Cleaner Production. 180, 876-887. Doi: 10.1016/j.jclepro.2018.01.089 
Yuan Z.W., Jun B., Moriguichigui Y.C. (2006). L'ecologia circolare: una nuova strategia di sviluppo in Cina. Journal of Industrial Ecology. 10, 4-8. Doi: 10.1162/108819806775545321

Zhao H., Gao Q., Wu Y., Wang Y., Zhu X. (2014). What affects green consumer behavior in China? A case study from Qingdao. Journal of Cleaner Production. 63, 143-151. Doi: 10.1016/j.jclepro.2013.05.021 